\title{
Thank you to our Reviewers
}

We are indebted to the expert referees who have reviewed submissions to the Canadian Journal of Neurological Sciences in 2014 (names in bold reviewed five or more papers). Their thoughtfulness and expertise have served our journal well.

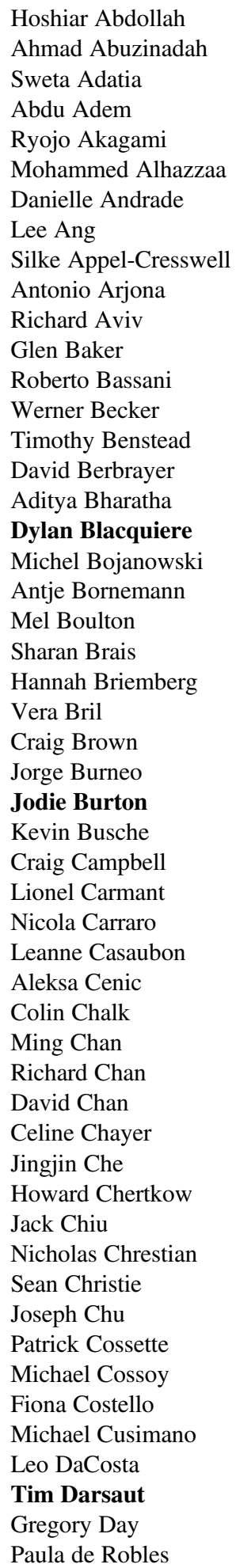

Nathan Deis

Marc Del Bigio

Linda De Meirleir

Michelle Demos

Sandrine

De Ribaupierre

Yan Deschaintre

Rajat Dhar

Esma Dilli

Dubravka Dodig

Tim Doherty

Joseph Dooley

Antoine Duquette

Alexander Easton

George Ebers

Michael Farrell

Alfonso Fasano

J Max Findlay

Daryl Fourney

Richard Fox

Susan Fox

Alex Fraser

Roger Freeman

Maria Frosini

Horvath Gabriella

Cynthia Gagnon

Renata Gallagher

Puja Garg

Thomas Geeraerts

Angela Genge

Amer Ghavanini

Elias Giraldo

Jonathan Glass

Teneille Gofton

Sharan Goobie

Alan Goodridge

Mayank Goyal

Candida Graham

Donald Griesdale

Gonzalo Guerra

Juan Guzman

Wael Haddara

Antoine Hakim

Hen Hallevi

William Halliday

Mark Hamilton

Michael Hill

Luke Hnenny

Matthew Hogan

David Holdsworth

Yoon-Ho Hong

Gabriella Horvath
Robin Hsiung

Xueqiang $\mathrm{Hu}$

Juliette Hukin

Shazam Hussein

Micheil Innes

Aaron Izenberg

Gerard Jansen

Mary Jenkins

Janine Johnston

M. Jones-Gotman

Hans Katzberg

Grover Kavita

Julia Keith

Tim Kennedy

Kurt Kimpinski

Andrew Kirk

Adam Kirton

Stephen Kish

Lawrence Korngut

Milica G. Kramberger

Jörg Kraus

Jacek Kurzepa

Anne-Louise

Lafontaine

Robert Jr Laforce

Anthony Lang

Sylvain Lanthier

Sandrine Larue

J.A. Leavitt

Ting-Yim Lee

Andrew Leung

Stan Leung

Yun-Man Li

Elan Louis

David MacDonald

Loch MacDonald

Matylda Machnowska

Chris MacKnight

Saadet Mahmutoglu

Todd Mainprize

Jennifer Mandzia

Bruno Maranda

Judith Marcoux

Tom Marotta

James Marriott

Shawn Marshall

Wayne Martin

Mario Masellis

Chuck Maxner

Patrick McDonald

Roger McKelvey

Richard McLachlan
Joseph Megyesi

Vivek Mehta

Daniel Mendelsohn

Bijoy Menon

Michelle Mezei

Tom Miller

Aleksandra Mineyko

Alim Mitha

Jeremy Moeller

Walter Montanera

Carlos Morillo

Sarah Morrow

Dwight Moulin

Howard Mount

Renato Munhoz

T. Jock Murray

S. Terence Myles

Yannick Nadeau

Giovanni Neri

John Norris

Jonathan Norton

Kenkichi Nozaki

Megan O'Connell

Joel Oger

Cian O'Kelly

Robert Olson

Antonina Omisade

Andre Parent

Vivien Parker

Phyllis Paterson

Leema Reddy

Peddareddygari

David Pelz

James Perry

Michelle Ploughman

Chitra Prasad

Alain Proulx

William Pryse-Phillips

Julian Raiman

Venkatakrishna

Rajajee

Ali Rajput

Alex Rajput

Lawrence Richer

Angela Roberts

Tuppy Rogers

Pedro Rosa-Neto

Paul Sandor

Tejas Sankar

Bernard Harvey Sarnat

Martin Savard

Rick Schreiber
Sasa Sega

Mohammed Shamji

Michael Sharma

Christen Shoesmith

Ashfaq Shuaib

Joseph Silvaggio

Brian Silver

John Sinclair

Elizabeth Slow

Peter Soros

Julian Spears

Sylvia

Stockler-Ipsiroglu

Erwin Stolz

Grant Stotts

Antonio Strafella

Stephen Strother

Tobias Struffert

Oksana Suchowersky

Rachita Sumbria

Rick Swartz

Sean Symons

Peter Tai

Donatella Tampieri

Jose Tellez-Zenteno

Eyad Al Thenayan

Charlie Thompson

Bernd Tomandl

Cory Toth

Brian Toyota

Tony Traboulsee

Jenny P. Tsai

John Turnbull

Maria Valencia

Martin Veilleux

Shannon Venance

Anand Viswanathan

Mehta Vivek

Simon Walling

Chun-Bo Wang

Theodore Wein

Matt Wheatley

Shawn Whitehead

Elaine Wirrell

Daniel Yavin

Keir Yong

Minming Zhang

Jingya Zhang

Yonghong Zhang 